\title{
Evaluation of Auto-segmentation for External Radiotherapy Planning Structures:A Part of Deep Learning Based Workflow in Cervical Cancer
}

Jiahao Wang

Women's Hospital, School of Medicine, Zhejiang University

Yuanyuan Chen

Women's Hospital, School of Medicine, Zhejiang University

Hongling Xie

Women's Hospital, School of Medicine, Zhejiang University

Lumeng Luo

Women's Hospital, School of Medicine, Zhejiang University

Qiu Tang ( $\nabla$ qiutang@zju.edu.cn )

Women's Hospital, School of Medicine, Zhejiang University

\section{Research Article}

Keywords: Deep learning, Auto-segmentation, Cervical cancer, Radiotherapy

Posted Date: March 2nd, 2022

DOI: https://doi.org/10.21203/rs.3.rs-1390719/v1

License: (9) This work is licensed under a Creative Commons Attribution 4.0 International License.

Read Full License 


\section{Abstract}

Objective:Deep learning (DL) based approach aims to construct a full workflow solution for cervical cancer with external radiotherapy (EBRT) and brachytherapy (BT).The purpose of this study was to evaluate the accuracy of EBRT planning structures derived from DL based auto-segmentation which would be a part implemented in the workflow of cervical cancer.

Methods: Clinical target volumes (CTVs) and organs at risk (OARs) of 75 cervical cancer patients were manually delineated by senior radiation oncologists and auto-segmented by DL based method.The accuracy of DL based auto-segmented contours were evaluated using geometric and dosimetric metrics including dice similarity coefficient (DSC), $95 \%$ hausdorf distance (95\%HD),jaccard coefficient (JC) and dose-volume index (DVI).The correlation between geometric metrics and dosimetric difference was performed by Spearman's correlation analysis.

Results:The DL based auto-segmentation generated similar geometric performance in right kidney,left kidney,bladder,right femoral head and left femoral head with mean DSC of $0.88-0.93,95 \%$ HD of $1.03-$ $2.96 \mathrm{~mm}$ and $\mathrm{JC}$ of $0.78-0.88$. Wilcoxon's signed-rank test indicated significant dosimetric differences between manual and DL based auto-segmentation in CTV,spinal cord and pelvic bone $(P<0.001)$. A strong correlation between the mean dose of pelvic bone and its $95 \% H D(R=0.843, P=0.000)$ was found in Spearman's correlation analysis, and the remaining structures showed weak link between dosimetric difference and all of geometric metrics.

Conclusion:Auto-segmentation achieved a satisfied agreement for most EBRT planning structures,although the dosimetric consistency of CTV was a concern. DL based auto-segmentation was an essential component in cervical cancer workflow which would generate the accurate contouring.

\section{Introduction}

External radiotherapy (EBRT) and brachytherapy (BT) are both the critical treatment modalities for cervical cancer with early and locally advanced stages. The delineation of clinical target volumes (CTVs) and organs at risk (OARs) is the first step and important task that may affect the clinical outcomes in cervical cancer radiotherapy[1-3].Indeed,manual contouring of these planning structures is such a laborintensive part of the workflow and maybe inaccurate[4-6].The workload pressures and most errors could be avoided if a rapid and accurate auto-segmented methods were available. With the development of machine learning $(M L)$,particularly the advent of deep learning $(D L)$ represented by convolutional neural networks (CNNs),auto-segmented tasks are thought to provide excellent assistance and superior results[7-10].

The U-Net model used for auto-segmentation of OARs in cervical cancer obtained highly consistent with those of expert contouring which was assessed by radiation oncologists[11].The DpnUNet model applied to CTV segmentation in cervical cancer achieved an acceptable clinical results with the mean dice similarity coefficient (DSC) of 0.86[12].As a novel technique,however, there are still some limitations with 
uncommon clinical practice[13].In fact,DL based methods are usually to generate the expected outcomes because the validated and tested datasets are typically related to the training datasets.Therefore,the quality and reliability of DL models should be further verified using an independent cohort in the process of cervical cancer radiotherapy.

The geometric metrics and subjective assessment are always chosen as the standard analysis indicators of contour comparison[14-16].A few studies have reported the relationship between auto-segmentation and dosimetry in head and neck which proved more accurate auto-segmentation carried out smaller dosimetric differences[17].However,whether or not the differences of DL based auto-segmentation would affect the clinical relevance of cervical cancer is rarely mentioned.

The purpose of this study used geometric and dosimetric metrics to evaluate the accuracy of DL based auto-segmentation and focus on the question of whether DL based approach could generate precise dosimetric endpoints compared to standard manual contours in a real-world independent cohort of cervical cancer patients.

\section{Methods And Materials}

\section{Experiments}

The work flowchart of this study is illustrated in Figure 1.Briefly,the evaluation was divided into three sections.Firstly,the accuracy of DL based auto-segmentation was assessed using geometric metrics.Secondly,the dosimetric differences were compared between standard manual contours and auto-segmented contours form original EBRT plans.Finally, the correlation was explored followed by geometric and dosimetric metrics.

\section{Clinical datasets}

This study consisted of 75 cervical cancer patients who received EBRT at our department between August 2021 and December 2021. All patients were diagnosed with FIGO stage IA2-IVB and histology G1-G3, treated with prescription dose of $45 \mathrm{~Gy}-50.4 \mathrm{~Gy}$ (1.8Gy/fraction).The average age \pm standard deviation of these patients was $55.60 \pm 13.35$ years old.For each patient,the contrast agent was required to intravenously inject before CT scanning,meanwhile,the CT images were covered from the lower lumbar spine to the whole pelvic cavity and reconstructed with $512 \times 512$ matrix size and $5 \mathrm{~mm}$ slice thickness using a Philips Brilliance Big Bore CT scanner system (Philips Healthcare,Best, the Netherlands).

CTVs delineation of 75 patients were defined manually by junior radiation oncologists including entire cervix, uterus, bilateral parametria,upper half of vagina, and lymph nodes following the guideline of Radiation Therapy Oncology Group (RTOG) protocol[18]. Relevant OARs included for EBRT plans were spinal Cord,lift kidney (Kidney L),right kidney (Kidney R),bladder,lift femoral Head (Femoral Head L),right femoral Head (Femoral Head R),pelvic bone,rectum, and small intestine.The EBRT planning structures were performed on the Pinnacle Treatment Planning System (Pinnacle, V9.16.2, Philips Corp, Fitchburg, 
WI, USA). All of the manual contours were reviewed and approved by senior radiation oncologists specialized in cervical cancer to generate the standard delineation.

\section{Deep learning based auto-segmentation}

We introduced a deep learning model based on CNN [19] to segment the CTVs and OARs for cervical cancer patients. Three hundred patients diagnosed with cervical cancer who received radiotherapy were enrolled for training this model,and the clinical and training datasets were come form different hospitals in order to verify the robustness of CNN model.As shown in Figure 2, the network consists of three encoders and three decoders. The InProj was used to extract the features of medical image, and the OutProj performed the pixel-wise classification. Spatial down-sampling and up-sampling were performed by each encoder and each decoder.The skip connection was used to concatenate the encoder and decoder of the same level to facilitate the fusion of multi-layer features.This model is an end-to-end segmentation architecture that can predict pixel class labels in CT images.

\section{Geometric metrics}

The geometric accuracy of contours was compared using the Dice Similarity Coefficient (DSC), $95 \%$ Hausdorff Distance (HD) and Jaccard Coefficient (JC).DSC and JC describe the relative overlap between segmentation $A$ and $B$. HD is used to quantify the $3 D$ distance between two segmentation surfaces. The $95 \% \mathrm{HD}$ is the distance that indicates the largest surface-to-surface separation among the closest $95 \%$ of surface points. The definitions are as follows:

$$
\begin{aligned}
& D S C=2|A \cap B| /(|A|+|B|) \\
& H D=\max (h(A, B), h(B, A)), h(A, B)=\max _{b \in B}\left(\min _{a \in A} \| a-b \mid\right) \\
& J C=|A \cap B| /|A \cup B|
\end{aligned}
$$

For the complete overlap, the value of $\mathrm{HD}$ is 0 , and the values of DSC and JC are 1 . For the incomplete overlap, the value of HD is large, and the values of DSC and JC are close to 0. In order to verify the recognition performance of $D L$ based model in boundary of segmentation,no cropping of the superior or inferior borders for contours was performed for this study particularly in spinal cord,femoral head and pelvic bone.

\section{Dosimetric metrics}

The EBRT plans were calculated and optimized with these standard manual contours by using Pinnacle Treatment Planning System.Table 1 was presented the constraints and dosimetric metrics.For CTV,we mainly focused on $D_{\text {mean }}$ and $V_{100 \%}$.For serial organs and parallel organs, we mainly focused on $D_{\text {max }}$ and $D_{\text {mean }}$,respectively. $D_{\text {mean }}$ and $D_{\max }$ are defined as the average dose and maximum dose of structures receiving. $\mathrm{V}_{100}$ is defined as the volume of CTV receiving $100 \%$ prescription dose. 


\section{Table 1}

The constraints and dosimetric metrics for EBRT planning structures.

\begin{tabular}{|c|c|c|}
\hline Structures & Constraints & Dosimetric metrics \\
\hline \multirow[t]{2}{*}{ CTV } & D $_{99 \%}>$ Prescription, & $D_{\text {mean }}, V_{100}$ \\
\hline & $D_{\max }<110 \%$ Prescription & \\
\hline Spinal Cord & $\mathrm{D}_{\max }<4000 \mathrm{cGy}$ & $D_{\max }$ \\
\hline Kidney L & $\mathrm{D}_{\text {mean }}<1200 \mathrm{cGy}$ & $\mathrm{D}_{\text {mean }}$ \\
\hline Kidney R & $\mathrm{D}_{\text {mean }}<1200 \mathrm{cGy}$ & $\mathrm{D}_{\text {mean }}$ \\
\hline \multirow[t]{2}{*}{ Bladder } & $\mathrm{D}_{50 \%}<100 \%$ Prescription & $D_{\text {mean }}$ \\
\hline & $\mathrm{D}_{0.03 c c}<110 \%$ Prescription & \\
\hline Femoral Head L & $\mathrm{D}_{15 \%}<3000 \mathrm{cGy}$, Mean dose $<2000 \mathrm{cGy}$ & $\mathrm{D}_{\text {mean }}$ \\
\hline Femoral Head R & $\mathrm{D}_{15 \%}<3000 \mathrm{cGy}$,Mean dose $<2000 \mathrm{cGy}$ & $\mathrm{D}_{\text {mean }}$ \\
\hline Pelvic Bone & $\mathrm{D}_{\text {mean }}<3000 \mathrm{cGy}$ & $\mathrm{D}_{\text {mean }}$ \\
\hline \multirow[t]{2}{*}{ Rectum } & $D_{50 \%}<100 \%$ Prescription & $D_{\text {mean }}$ \\
\hline & $\mathrm{D}_{0.03 c c}<110 \%$ Prescription & \\
\hline \multirow[t]{2}{*}{ Small intestine } & $\mathrm{D}_{30 \%}<100 \%$ Prescription & Not evaluated \\
\hline & $\mathrm{D}_{0.03 c c}<110 \%$ Prescription & \\
\hline
\end{tabular}

\section{Statistical analysis}

IBM SPSS Statistics software (version 19.0,IBM Inc., Armonk, NY, USA) and Python software (version 3.6.5,Anaconda Inc.) were used for statistical analysis, where mean \pm standard deviation (SD) was used for presenting and summarizing the results. The Wilcoxon's paired nonparametric signed-rank test was performed to compare the variables between the manual and DL based methods. $P<0.05$ indicates that the difference is statistically significant. The correlations between geometric metrics and dosimetric difference were evaluated with Spearman's correlation analysis. The $\Delta$ dose was used to define the dosimetric difference between two methods.

\section{Results}


The geometric accuracy of the DL based auto-segmentation for EBRT planning structures is summarized in figure 3.Automatic delineation produced a relatively good result for the CTV with average DSC value of $0.77 \pm 0.03,95 \% \mathrm{HD}$ of $5.81 \pm 1.83 \mathrm{~mm}$ and $\mathrm{JC}$ of $0.62 \pm 0.04$. The right kidney, left kidney,bladder, right femoral head and left femoral head were generated the similar geometric performance between two methods with average DSC value of $0.88-0.93,95 \% \mathrm{HD}$ of $1.03-2.96 \mathrm{~mm}$ and $\mathrm{JC}$ of $0.78-0.88$. The quality of the automatically generated pelvic bone was barely satisfactory with average DSC value of $0.65 \pm 0.05,95 \% \mathrm{HD}$ of $18.14 \pm 9.77 \mathrm{~mm}$ and $\mathrm{JC}$ of $0.49 \pm 0.05$.

Examples of delineations and dose distributions from manual and DL based auto-segmented methods are illustrated in Figure 4. The comparisons of dosimetric parameters between two methods using Wilcoxon's paired nonparametric signed-rank test are presented in Table 2. No significant dosimetric differences were found except for CTV,spinal cord and pelvic bone $(P<0.001)$. For all of the OARs, both the manual and automatic delineation were able to meet the clinical dose constraints. However,the dosevolume index (DVI) of CTV was hard to meet the clinical requirements with $\mathrm{V}_{100}(\%)$ of $94.27 \pm 1.86$ ( $D_{99 \%}>$ Prescription).

\section{Table 2}

Dosimetric metrics of manual and DL based auto-segmented delineations in the original clinical treatment plans.

\begin{tabular}{|c|c|c|c|c|c|}
\hline Structure & $\begin{array}{l}\text { Dosimetric } \\
\text { parameters }\end{array}$ & $\begin{array}{l}\text { Manual } \\
\text { delineation }\end{array}$ & $\begin{array}{l}\text { Automatic } \\
\text { delineation }\end{array}$ & Z & $\mathrm{P}$ \\
\hline & & \multicolumn{4}{|c|}{ mean \pm standard deviation } \\
\hline \multirow[t]{2}{*}{ CTV } & $\mathrm{D}_{\text {mean }}(\mathrm{cGy})$ & $5058.71 \pm 191.85$ & $4972.37 \pm 194.18$ & -7.53 & $<0.001$ \\
\hline & $\mathrm{V}_{100}(\%)$ & $99.98 \pm 0.02$ & $94.27 \pm 1.86$ & -7.53 & $<0.001$ \\
\hline Spinal Cord & $\mathrm{D}_{\max }(\mathrm{cGy})$ & $3270.17 \pm 259.72$ & $3616.79 \pm 565.83$ & -4.00 & $<0.001$ \\
\hline Kidney L & $\mathrm{D}_{\text {mean }}(\mathrm{cGy})$ & $175.41 \pm 320.37$ & $171.29 \pm 306.65$ & -1.67 & 0.096 \\
\hline Kidney R & $\mathrm{D}_{\text {mean }}(\mathrm{cGy})$ & $205.68 \pm 318.12$ & $201.80 \pm 308.71$ & -0.99 & 0.323 \\
\hline Bladder & $\mathrm{D}_{\text {mean }}(\mathrm{cGy})$ & $4345.07 \pm 263.11$ & $4338.67 \pm 270.32$ & 0.95 & 0.342 \\
\hline $\begin{array}{l}\text { Femoral Head } \\
\text { L }\end{array}$ & $\mathrm{D}_{\text {mean }}(\mathrm{cGy})$ & $1930.73 \pm 74.68$ & $1928.89 \pm 97.26$ & -0.48 & 0.631 \\
\hline $\begin{array}{l}\text { Femoral Head } \\
\text { R }\end{array}$ & $\mathrm{D}_{\text {mean }}(\mathrm{cGy})$ & $1897.44 \pm 75.49$ & $1901.13 \pm 84.13$ & -1.24 & 0.085 \\
\hline Pelvic Bone & $\mathrm{D}_{\text {mean }}(\mathrm{cGy})$ & $2802.99 \pm 129.23$ & $2968.00 \pm 160.85$ & -7.48 & $<0.001$ \\
\hline Rectum & $\mathrm{D}_{\text {mean }}(\mathrm{cGy})$ & $4490.24 \pm 252.17$ & $4523.57 \pm 248.61$ & -1.61 & 0.108 \\
\hline
\end{tabular}


Table 3 shows the results of Spearman's correlation analysis between three geometric metrics and dosimetric differences ( $\Delta$ dose). No structures showed strong correlation except for the $\Delta D_{\text {mean }}$ of pelvic bone and its $95 \% \mathrm{HD}(\mathrm{R}=0.843, \mathrm{P}=0.000)$, and the correlation heatmap was used to further prove the weak link between all of the dosimetric difference and its geometric metrics in the remaining EBRT planning structures (figure 5).

\section{Table 3}

The correlation between geometric metrics and dosimetric differences. 


\begin{tabular}{|c|c|c|c|}
\hline Structure & $\Delta$ Dose & Geometric metrics & Correlation analysis \\
\hline \multirow[t]{3}{*}{ CTV } & $\Delta \mathrm{D}_{\text {mean }}$ & DSC & $R=-0.198, P=0.089$ \\
\hline & & $95 \% \mathrm{HD}$ & $R=0.089, P=0.087$ \\
\hline & & $\mathrm{JC}$ & $R=-0.195, P=0.093$ \\
\hline \multirow[t]{3}{*}{ CTV } & $\Delta \mathrm{V}_{100}(\%)$ & DSC & $R=-0.245, P=0.034$ \\
\hline & & $95 \% \mathrm{HD}$ & $R=0.180, P=0.123$ \\
\hline & & $\mathrm{JC}$ & $R=-0.245, P=0.034$ \\
\hline \multirow[t]{3}{*}{ Spinal Cord } & $\Delta \mathrm{D}_{\max }$ & DSC & $R=0.047, P=0.688$ \\
\hline & & $95 \% \mathrm{HD}$ & $R=0.046, P=0.694$ \\
\hline & & $\mathrm{JC}$ & $R=0.043, P=0.711$ \\
\hline \multirow[t]{3}{*}{ Kidney L } & $\Delta \mathrm{D}_{\text {mean }}$ & DSC & $R=-0.076, P=0.518$ \\
\hline & & $95 \% \mathrm{HD}$ & $R=0.162, P=0.166$ \\
\hline & & $\mathrm{JC}$ & $R=-0.074, P=0.528$ \\
\hline \multirow[t]{3}{*}{ Kidney R } & $\Delta \mathrm{D}_{\text {mean }}$ & DSC & $R=-0.361, P=0.001$ \\
\hline & & $95 \% \mathrm{HD}$ & $R=0.379, P=0.001$ \\
\hline & & $\mathrm{JC}$ & $R=-0.354, P=0.002$ \\
\hline \multirow[t]{3}{*}{ Bladder } & $\Delta \mathrm{D}_{\text {mean }}$ & DSC & $R=-0.644, P=0.000$ \\
\hline & & $95 \% \mathrm{HD}$ & $R=0.601, P=0.000$ \\
\hline & & $\mathrm{JC}$ & $R=-0.646, P=0.000$ \\
\hline \multirow[t]{3}{*}{ Femoral Head L } & $\Delta D_{\text {mean }}$ & DSC & $R=-0.341, P=0.003$ \\
\hline & & $95 \% \mathrm{HD}$ & $R=0.225, P=0.052$ \\
\hline & & $\mathrm{JC}$ & $R=-0.349, P=0.002$ \\
\hline \multirow[t]{3}{*}{ Femoral Head R } & $\Delta \mathrm{D}_{\text {mean }}$ & DSC & $R=-0.014, P=0.902$ \\
\hline & & $95 \% \mathrm{HD}$ & $R=0.095, P=0.418$ \\
\hline & & $\mathrm{JC}$ & $R=-0.015, P=0.899$ \\
\hline \multirow[t]{3}{*}{ Pelvic Bone } & $\Delta \mathrm{D}_{\text {mean }}$ & DSC & $R=-0.588, P=0.000$ \\
\hline & & $95 \% \mathrm{HD}$ & $R=0.843, P=0.000$ \\
\hline & & $\mathrm{JC}$ & $R=-0.589, P=0.000$ \\
\hline
\end{tabular}




\begin{tabular}{cll} 
Rectum $\Delta D_{\text {mean }}$ & DSC & $R=0.054, P=0.648$ \\
& $95 \% H D$ & $R=-0.082, P=0.482$ \\
\hline JC & $R=0.055, P=0.641$
\end{tabular}

\section{Discussion}

Modern radiotherapy has become a systematized and programmed process resulting in a nearly reliance on human-machine interactions with the development of mechanical technology and computer science.Meanwhile,the growth of Artificial intelligence (Al) has the potential possibilities to change the way of radiation oncology because of its recognition and analysis in complex medical data.Various studies have investigated the advantages of Al based method during each stage of radiotherapy,such as Al platforms might improve the efficiency and quality of automated segmentation[20-22],predict and optimize the radiation dose of the targets[23,24],provide the clinical decision of radiation toxicities[25], and build the robust models to manage the treatment outcomes[26,27]. However,these studies were always fragmented and we should establish the complete radiotherapy workflow using $\mathrm{Al}$ technology with validating every step for the real-world cohort.

Delineations of CTV and OARs are an essential step for precise delivery[28] which would affect the overall survival in the radiotherapy treatment planning process,even in standardizing clinical trials[29].However, the manual process always suffers from inter- and intra-observer variability in structure delineations.Automatic contouring of structures is highly desired in radiotherapy because of the minimized variability. The purpose of this study is to compare the performance of DL based auto[1]segmentation against standard contours from senior radiation oncologists on independent dataset.

We observed that DL based model generated structures with average DSC of 0.77 for the CTV, 0.74 for the spinal cord, 0.93 for the left and right kidney, 0.91 for the bladder, 0.88 for the left and right femoral head, 0.65 for the pelvic bone,and 0.71 for the rectum,respectively.For all the structures,the kidney,bladder and femoral head presented the superior performance because of relatively constant volume among the different patients. The mean $95 \% \mathrm{HD}$ value of CTV was $5.81 \mathrm{~mm}$, which was comparable to $5.34 \mathrm{~mm}$ [12] by the DpnUNet model.The results seemed to indicate a strong concordance between the DL based model and experts for CTV segmentation. However,the boundaries of the rectum and spinal cord in cervical cancer were not clear (the resolution of soft tissue in CT images was deficient and we didn't modify the superior or inferior borders), the delineations generated by DL based model were always been overestimated or underestimated compared with standard contours. Meanwhile,the accuracy of pelvic bone was worse than reported by Liu [11](DSC=0.85) and Rhee[30](DSC=0.93). This finding might have been caused by the difference of training datasets between these networks.In this study,we didn't assess the small intestine because the location of the small intestine in CT images was different from the location during EBRT process. Indeed,small intestine is an important organ for dosimetric evaluation 
especially in the EBRT combined with high-dose rate BT for cervical cancer, and the DL based performance of small intestine would be included in our further study with "dose prediction".

The quality of auto segmented contours cannot be determined only by geometric values which was reported by Kaderka [31], and few studies have focused on dosimetric impact on the automatic CTV delineations for cervical cancer radiotherapy.For CTV dosimetric metrics, the most significant dose difference was $V_{100}$ with $94.27 \%$ for $D L$ based model and $99.98 \%$ for standard contour $(P<0.001)$, and the original dose distribution showed poor results in automatic CTV segmentation(Fig.5).These data indicated the final CTV segmentation generated by DL based model remains necessary to be reviewed by senior radiation oncologists rather than superior geometric values.For OARs dosimetric metrics,no significant dosimetric differences were found except for spinal cord and pelvic bone $(P<0.001)$. Point dose such as $D_{\max }$ in spinal cord was sensitive to the range of the segmentation in radiotherapy which means the performance of identifying boundaries in DL based model should be improved.

The heatmap of Spearman's correlation analysis showed that there was no clear strong relationship between geometric metrics and dosimetric differences for most structures(Fig.5).The only strong correlation was shown for the mean dose of pelvic bone and its $95 \% H D(R=0.843, P=0.000)$. This phenomenon cloud be explained that the dosimetric differences were generated by random noise because of the similar delineation between two methods such as kidney and bladder.Otherwise,the weak link was caused by the segmented reproducibility of DL based model such as CTV and femoral head.However,significant correlation between geometric metrics and dosimetric differences could still be observed due to the inaccurate delineation such as pelvic bone.

In this work,we investigated the performance of DL[1]based auto segmentation in cervical cancer for patients treated with EBRT.Indeed,as an assisted and efficient tool,automatic approach would relieve physicians from the labor-intensive tasks as well as increase the accuracy and reproducibility of structure delineation.Instead of incorporating a prior knowledge into the process of segmentation that describe as atlas-based segmentation (ABS)[32],DL based auto segmentation explores the informative representations in a self-learning algorithm and utilizes hierarchical layers of extracted abstraction to accomplish high-level tasks efficiently.Furthermore, in spite of the superior performance of DL based methods on algorithm, the studies are confined mostly to the field of segmentation rather than to establish the workflow solution which have been mentioned above.In other words,DL based methods could play an important role in the complete process of radiotherapy such as "dose prediction", "toxic prediction", "efficacy prediction",etc, segmentation/ "delineation prediction" is only a part of this workflow.Certainly,this work was focus on the question of segmented accuracy which would be a basic part implemented in the workflow of cervical cancer radiotherapy.

Several limitations still exist in our study.First,this work was lack of subjective assessment such as radiation oncologist evaluation or Turing imitation test[33].Second,the diversity of CT scanner machines,image acquisition protocols,standard contouring,and even tumor staging hampered meaningful comparison of our results with other CNN models. Overall,increasing the amount of training 
data from different centers using different techniques could make the DL based model more robust, improving the segmentation accuracy.

\section{Conclusion}

This study has demonstrated through both geometric and dosimetric metrics that a DL based autosegmentation can achieve clinically acceptable contours for most of the EBRT planning structures in cervical cancer patients,although the dosimetric consistency of CTV was a concern. Automatic delineation will be an essential component in cervical cancer workflow which would generate the accurate contouring.

\section{Declarations}

\section{Data availability}

The datasets used and/or analyzed during the current study available from the corresponding author on reasonable request.

\section{References}

[1]Eminowicz G,Rompokos V,Stacey C,et al.The dosimetric impact of target volume delineation variation for cervical cancer radiotherapy.Radiother Oncol.2016;120:493-499.

https://doi.org/10.1016/j.radonc.2016.04.028

[2]Dutta SW,Trifiletti DM,Pugh KJ,et al.Integration of MRI target delineation into rapid workflow cervical cancer brachytherapy: Impact on clinical outcomes.J Med Imaging Radiat Oncol. 2018;62:716725.https://doi.org/10.1111/1754-9485.12763.

[3]Weiss E,Richter S,Krauss T,et al.Conformal radiotherapy planning of cervix carcinoma: differences in the delineation of the clinical target volume. A comparison between gynaecologic and radiation oncologists. Radiother Oncol. 2003;67:87-95.

https://doi.org/ 10.1016/s0167-8140(02)00373-0.

[4]Vorwerk $\mathrm{H}$,Zink $\mathrm{K}$,Schiller R,et al. Protection of quality and innovation in radiation oncology: the prospective multicenter trial the German Society of Radiation Oncology (DEGRO-QUIRO study).Strahlentherapie und Onkol. 2014;190:433-443.https://doi.org/10.1007/s00066-014-0634-0.

[5]Chen KQ,Chen WJ,Ni X,et al.Systematic evaluation of atlas-based auto segmentation (ABAS) software for adaptive radiation therapy in cervical cancer.China J Radio Med Prot. 2015;35:111113.https://doi.org/10.3760/cma.j.issn.0254-5098.2015.02.008. 
[6]Ford E,Conroy L,Dong L,et al.Strategies for effective physics plan and chart review in radiation therapy: report of AAPM Task Group 275.Med Phys. 2020;47:236-272. https://doi.org/10.1002/mp.14030.

[7]Cardenas CE, Yang J, Anderson BM, Court LE, Brock KB. Advances in auto-segmentation. Semin Radiat Oncol. 2019;29:185-197.https://doi.org/10.1016/j.semradonc.2019.02.001.

[8]Lustberg T, van Soest J, Gooding M, et al. Clinical evaluation of atlas and deep learning based automatic contouring for lung cancer. Radiother Oncol. 2018;126(2):312-317.

https://doi.org/10.1016/j.radonc.2017.11.012.

[9]Sarıgül M,Ozyildirim BM,Avci M.Differential Convolutional Neural Network.Neural Networks 2019;116:279-287. https://doi.org/10.1016/j.neunet.2019.04.025.

[10]Kim N,Chang JS,Kim YB,et al.Atlas-Based Auto-Segmentation for Postoperative Radiotherapy Planning in Endometrial and Cervical Cancers.Radiat Oncol 2020;15:106.

https://doi.org/10.1186/s13014-020-01562-y.

[11] Liu Z, Liu X, Xiao B, et al. Segmentation of organs-at-risk in cervical cancer CT images with a convolutional neural network. Med Phys.2020;69:184-191.

https://doi.org/10.1016/j.ejmp.2019.12.008.

[12]Liu Z, Liu X,Guan H,et al. Development and Validation of a Deep Learning Algorithm for AutoDelineation of Clinical Target Volume and Organs at Risk in Cervical Cancer Radiotherapy. Radiother Oncol 2020;153:172-179. https://doi.org/10.1016/j.radonc.2020.09.060.

[13]Brouwer CL,Dinkla AM,Vandewinckele L,et al. Machine Learning Applications in Radiation Oncology:Current Use and Needs to Support Clinical Implementation. Phys Imaging Radiat Oncol 2020;16:144-148. https://doi.org/10.1016/j.phro.2020.11.002.

[14]Sharp G,Fritscher KD,Pekar V,et al.Vision 20/20: perspectives on automated image segmentation for radiotherapy. Med Phys 2014;41(5):1-13.https://doi.org/10.1118/1.4871620.

[15]Ren X,Xiang L,Nie D,et al.Interleaved 3D-CNNs for joint segmentation of small-volume structures in head and neck CT images. Med Phys 2018;45(5):2063-2075.

https://doi.org/10.1002/mp.12837.

Wong J, Fong A, McVicar N, et al. Comparing deep learning-based auto-segmentation of organs at risk and clinical target volumes to expert inter-observer variability in radiotherapy planning. Radiother Oncol. 2019;144:152-158.https://doi.org/1016/j.radonc.2019.10.019.

[17]Van Dijk LV, Van den Bosch L, Aljabar P, et al. Improving automatic delineation for head and neck organs at risk by Deep Learning Contouring. Radiother Oncol. 2020;142:115-123. 
https://doi.org/10.1016/j.radonc.2019.09.022.

[18] Small WJr.,Bosch WR,Harkenrider MM,et al. NRG Oncology/RTOG Consensus Guidelines for Delineation of Clinical Target Volume for Intensity Modulated Pelvic Radiation Therapy in Postoperative Treatment of Endometrial and Cervical Cancer: an Update. Int J Radiat Oncol Biol Phys 2021;109:413424.https://doi.org/10.1016/j.jijobp.2020.08.061.

[19] Long J, Shelhamer E, Darrell T. Fully convolutional networks for seman[1]tic segmentation. https://arxiv.org/abs/1411.4038v22015.

[20]Cardenas CE,McCarroll RE,Courtet LE,et al. Deep learning algorithm for auto-delineation of high-risk oropharyngeal clinical target volumes with built-in dice similarity coefficient parameter optimization function. Int. J. Radiat. Oncol. Biol. Phys. 2018;101:468-478.

https://doi.org/10.1016/j.jirobp.2018.01.114.

[21]Men K,Chen X,Zhang Y,et al. Deep deconvolutional neural network for target segmentation of nasopharyngeal cancer in planning computed tomography images. Front. Oncol.2017;7:315.

https://doi.org/10.3389/fonc.2017.00315.

[22]Mak RH,Endres MG,Paik JH,et al. Use of crowd innovation to develop an artificial intelligence-based solution for radiation therapy targeting. JAMA Oncol. 2019;5: 654.

https://doi.org/10.1001/jamaoncol.2019.0159.

[23]Nguyen $D$,Long $T$,Jia X,et al. A feasibility study for predicting optimal radiation therapy dose distributions of prostate cancer patients from patient anatomy using deep learning. Sci. Rep.2019; 9:1076.https://doi.org/ 10.1038/s41598-018-37741-x.

[24]Lou B,Doken S,Zhuang TL,et al. An image-based deep learning framework for individualising radiotherapy dose: a retrospective analysis of outcome prediction. Lancet Digital Health 2019; 1:136147.https://doi.org/10.1016/S2589-7500(19)30058-5.

[25]Lee $\mathrm{S}$, Kerns $\mathrm{S}, \mathrm{O}$ strer $\mathrm{H}$,et al. Machine learning on a genome-wide association study to predict late genitourinary toxicity after prostate radiation therapy. Int. J. Radiat. Oncol. Biol. Phys.2018; 101:128135.https://doi.org/10.1016/j.jirobp.2018.01.054.

[26]Deist TM,Jochems A,Oberije C,et al. Expert knowledge and data-driven Bayesian networks to predict post-RT dyspnea and 2-year survival. Radiother. Oncol 2016; 118:29-30.

https://doi.org/10.1016/S0167-8140(16)30060-3.

[27]Hong JC,Niedzwiecki D,Palta M,et al. Predicting emergency visits and hospital admissions during radiation and chemoradiation: an internally validated pretreatment machine learning algorithm. JCO Clin. 
Cancer Inform 2018;2:1-11.https://doi.org/10.1200/CCI.18.00037.

[28]Men K,Zhang T,Chen X,et al. Fully Automatic and Robust Segmentation of the Clinical Target Volume for Radiotherapy of Breast Cancer Using Big Data and Deep Learning. Phys Med 2018;50:1319.https://doi: 10.1016/j.ejmp.2018.05.006.

[29]Brade AM,Wenz F,Koppe F,et al.Radiation Therapy Quality Assurance (RTQA) of Concurrent Chemoradiation Therapy for Locally Advanced Non-Small Cell Lung Cancer in the PROCLAIM Phase 3 Trial. Int. J. Radiat. Oncol. Biol. Phys 2018;101:927-934.

https://doi:10.1016/j.ijrobp.2018.04.015.

[30]Rhee DJ,Jhingran A,Rigaud B,et al.Automatic contouring system for cervical cancer using convolutional neural networks.Phys Med 2020;47:5648-5658.https://doi.org/10.1002/mp.14467.

[31] Kaderka R, Gillespie EF, Mundt RC, et al. Geometric and dosimetric evalu[1]ation of atlas based autosegmentation of cardiac structures in breast cancer patients. Radiother Oncol

2019;131:215-220.https://doi.org/10.1016/j.radonc.2018.07.013.

[32]Iglesias JE, Sabuncu MR. Multi-atlas segmentation of biomedical images: a survey. Med Image Anal 2015;24:205-219. https://doi:10.1016/j.media.2015.06.012.

[33] Turing AM. Computing Machinery and Intelligence. Mind 1950; 433-460. https://doi:

10.1093/mind/LIX.236.433.

\section{Figures}




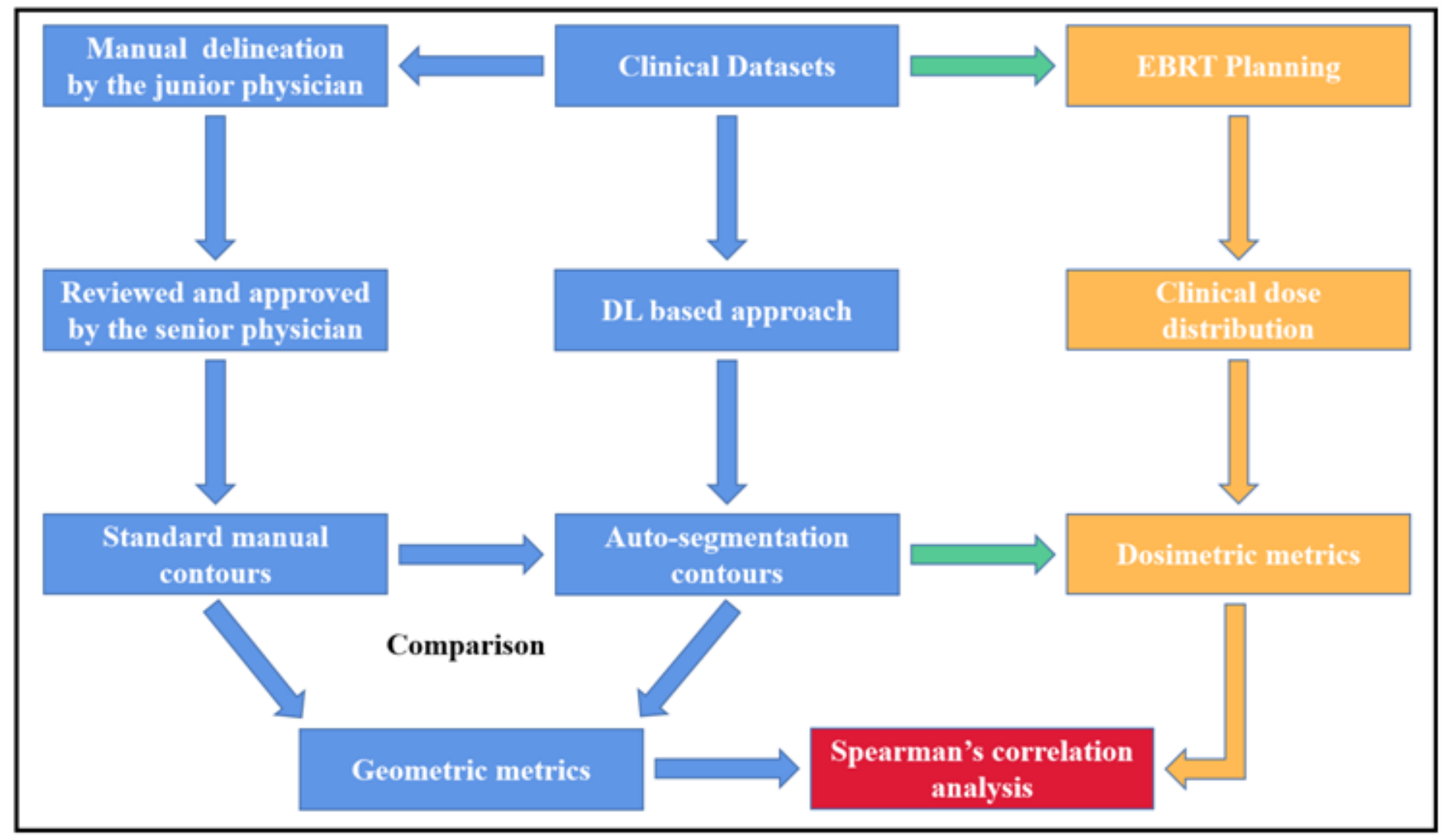

Figure 1

The flowchart of manual and DL based auto-segmentation evaluation experiment.

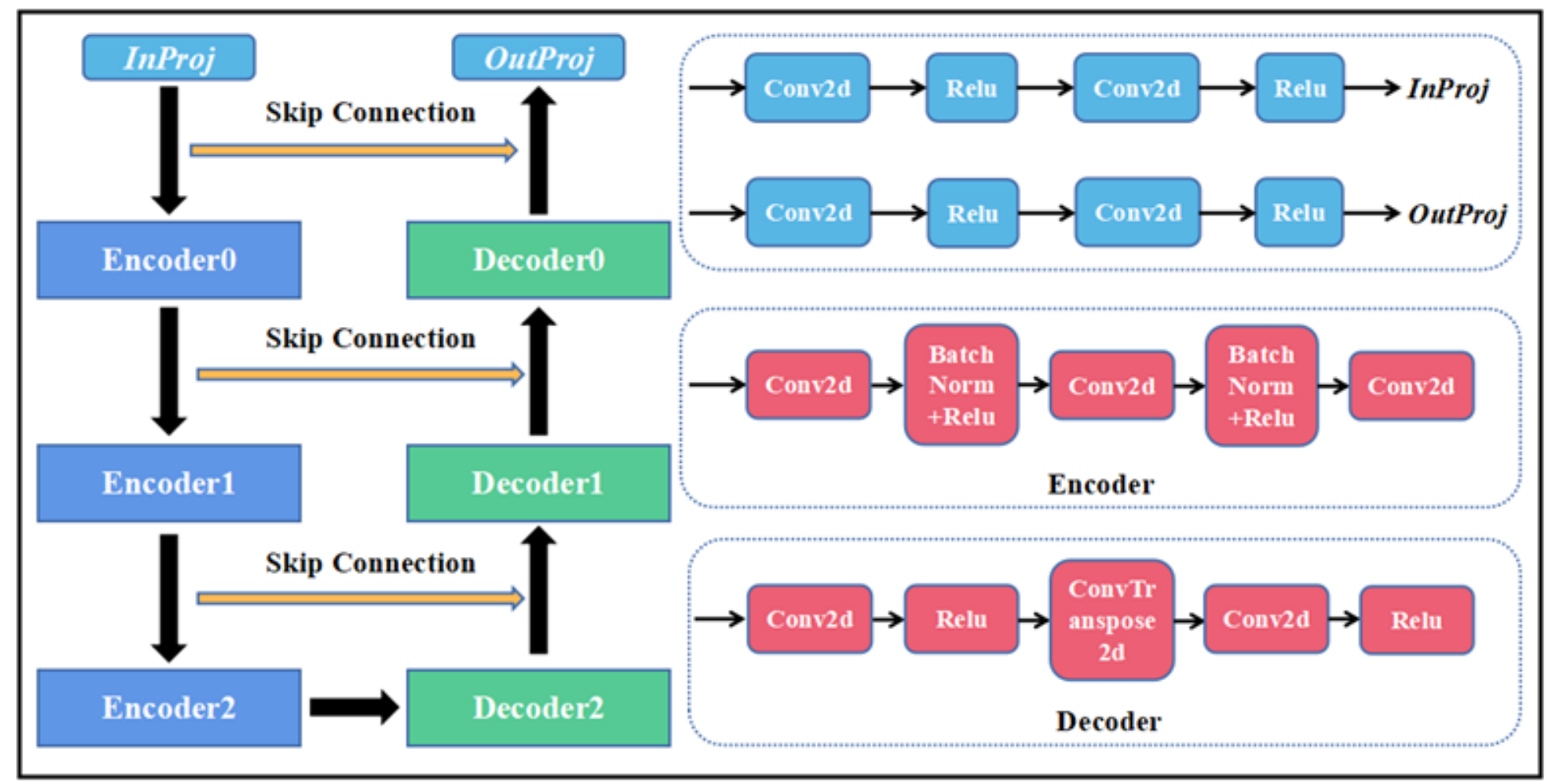

Figure 2 
Architecture of DL based automatic segmentation network.
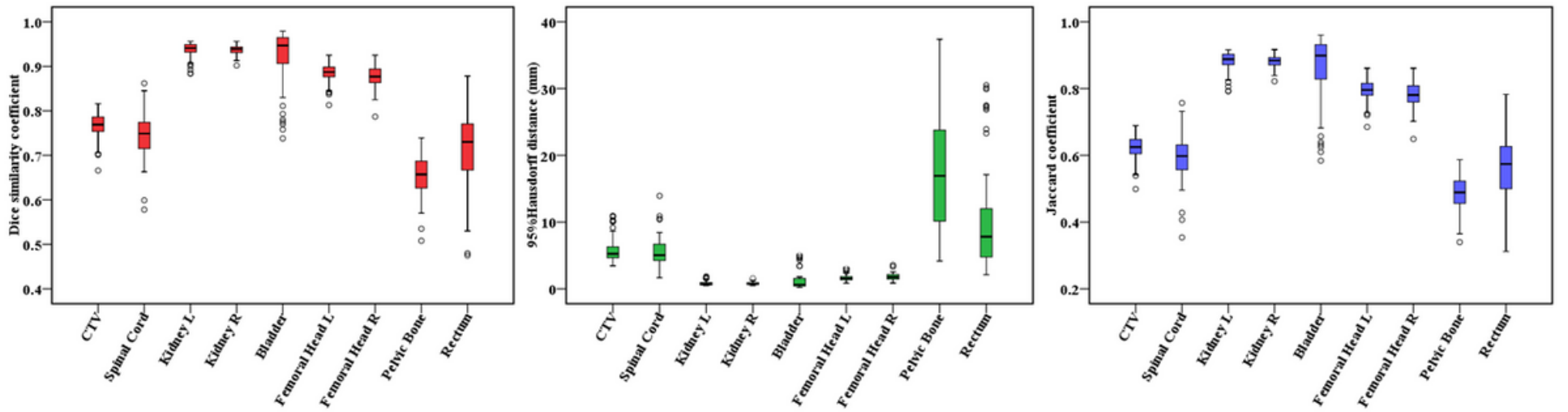

Figure 3

DSC,95\%HD and JC box plot from comparing DL based auto-segmented contours to standard contours for CTV and OARs.L=Left,R=Right.

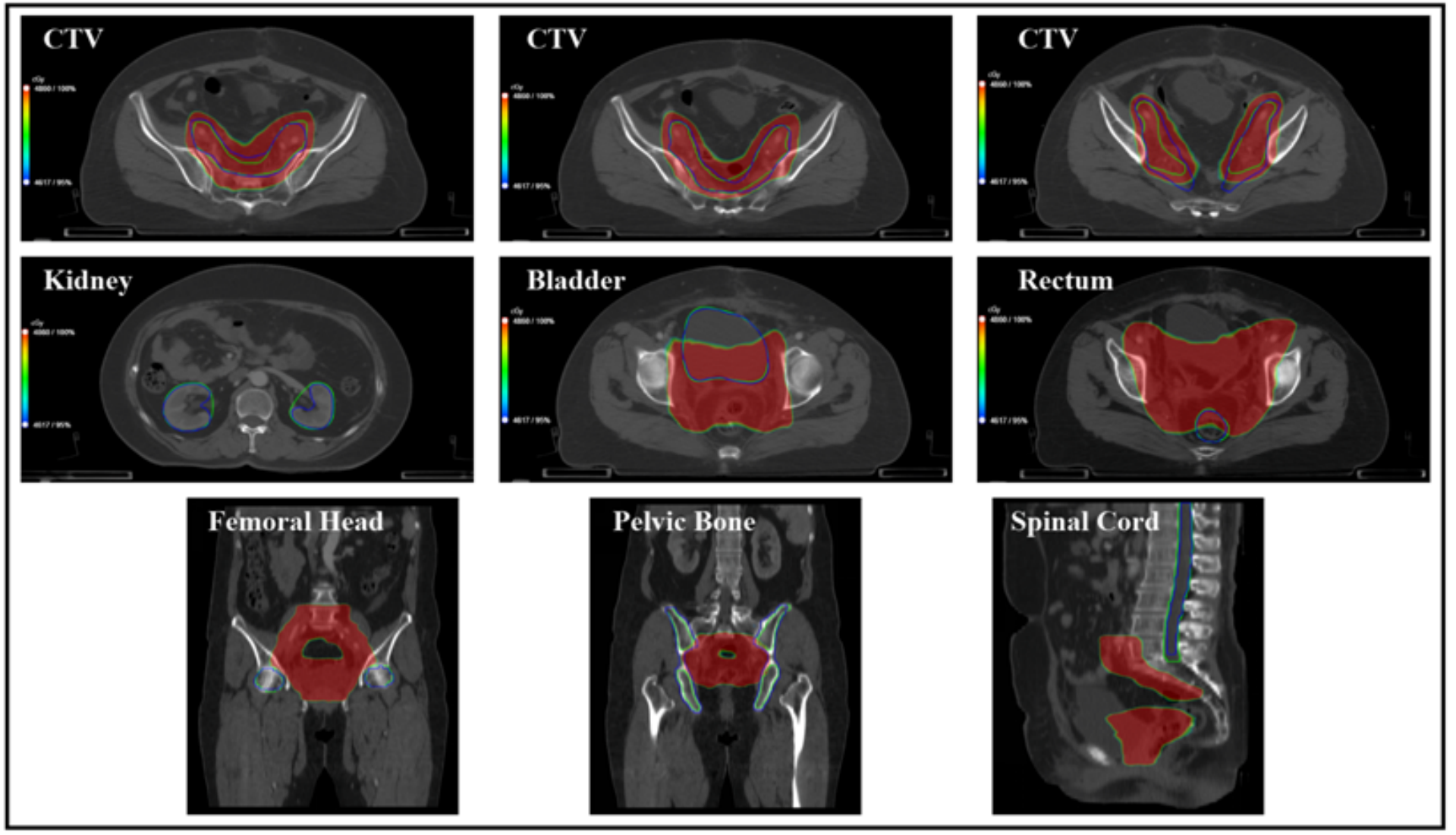

\section{Figure 4}

Results of delineations and dose distributions for CTV and OARs in CT slices.Green lines= Manual contours approved by the senior physician,Blue lines=DL based contours,Colourwash= dose distributions with the range of $95 \%$ prescription- $100 \%$ prescription. 


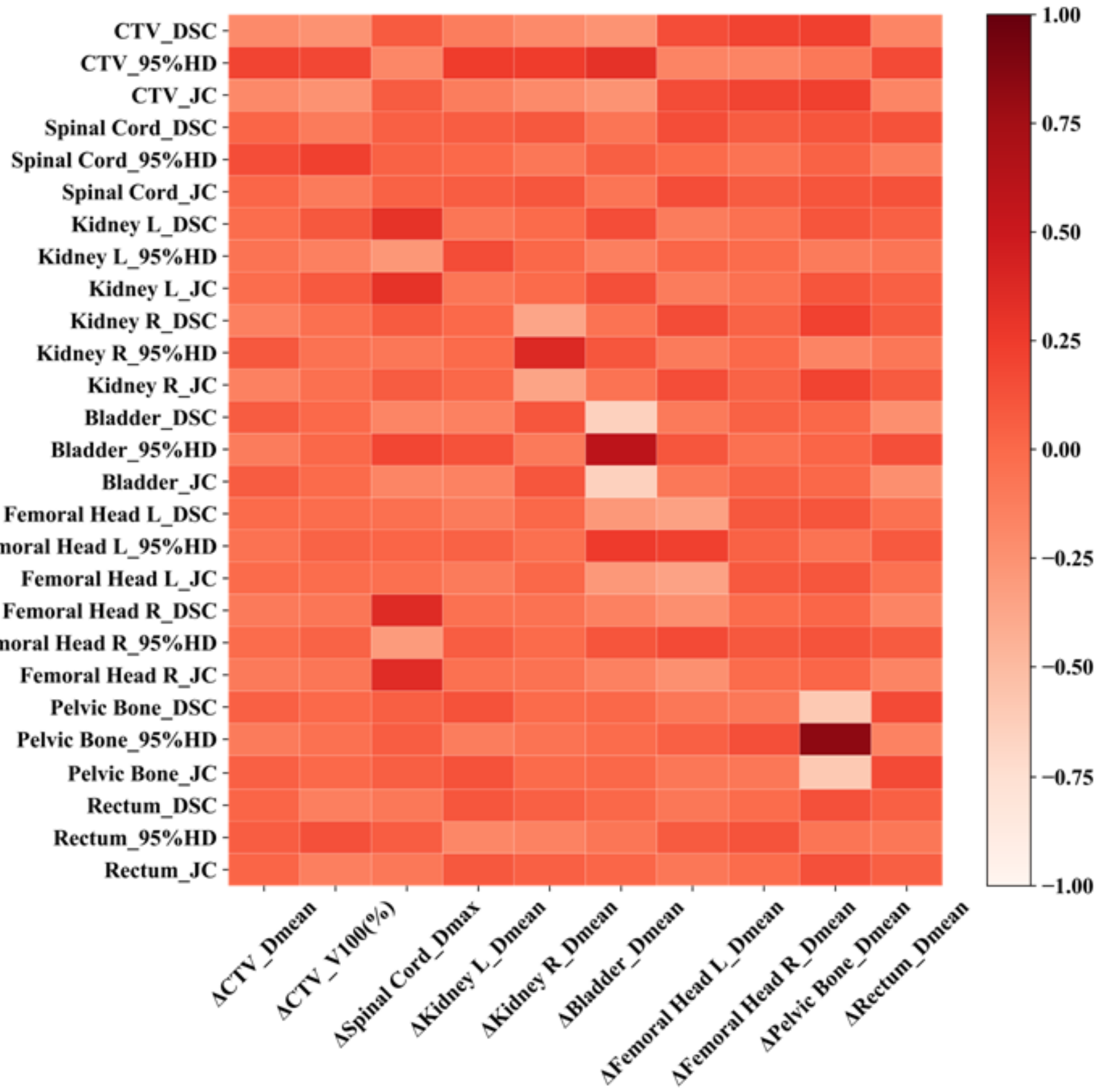

\section{Figure 5}

The heatmap of Spearman's correlation analysis between all the geometric metrics and dosimetric differences for EBRT planning structures. 